\title{
Contexte multilingue et langue nationale
}

Enseignement de l'arabe dans les classes de lycée au Maroc. Entretien avec MM. Elkhomsi Mohamed et Ahmid Mohamed

Michel Massacret et Roger Jeansoulin

\section{OpenEdition}

\section{Journals}

Édition électronique

URL : http://journals.openedition.org/ries/2869

DOI : 10.4000/ries.2869

ISSN : 2261-4265

Éditeur

Centre international d'études pédagogiques

\section{Édition imprimée}

Date de publication : 1 septembre 1998

Pagination : 61-65

ISSN : 1254-4590

\section{Référence électronique}

Michel Massacret et Roger Jeansoulin, «Contexte multilingue et langue nationale », Revue internationale d'éducation de Sèvres [En ligne], 19 | septembre 1998, mis en ligne le 18 avril 2013, consulté le 23 mars 2021. URL : http://journals.openedition.org/ries/2869 ; DOI : https://doi.org/ $10.4000 /$ ries. 2869

Ce document a été généré automatiquement le 23 mars 2021.

(c) Tous droits réservés 


\section{Contexte multilingue et langue nationale}

Enseignement de l'arabe dans les classes de lycée au Maroc. Entretien avec MM. Elkhomsi Mohamed et Ahmid Mohamed

Michel Massacret et Roger Jeansoulin

\section{Statut et finalités de l'arabe standard}

1 L'arabe standard ${ }^{1}$ a, au Maroc, le statut de langue nationale et de langue officielle, c'est aussi la langue du Coran, qui lie le monde arabe et le Maghreb. Cette langue, qui est à la fois langue officielle des institutions publiques, langue liturgique de l'Islam, langue de communication écrite et langue d'enseignement, n'est cependant pas la langue maternelle des élèves qui parlent chez eux l'arabe dialectal ou l'une des différentes variétés de la langue berbère. Ces deux langues qui ne sont pas standardisées et n'ont pas de statut reconnu, jouent le rôle de langues véhiculaires et tout particulièrement l'arabe dialectal.

2 Les finalités de son enseignement sont d'assurer la maîtrise de la langue écrite et de la communication médiatique, au sein de la communauté nationale et de la communauté arabe. Il s'agit également de développer la socialisation et le sentiment d'identité par la connaissance des réalités et de la culture aux différents niveaux, national, maghrébin, arabe et islamique.

\section{Les élèves et l'arabe standard}

\section{Problèmes d'apprentissage et d'enseignement}

3 Si le statut de l'arabe standard est clairement reconnu, son degré de maîtrise est très variable dans un pays multilingue où la langue maternelle des élèves est soit l'arabe dialectal, soit l'une des variétés de la langue berbère. 
4 L'apprentissage de l'arabe commence à partir de l'âge de 5 ans au msid (l'école coranique). Cet apprentissage, en rupture avec la langue parlée dialectale, est source de difficultés pour les enfants et a des aspects négatifs sur l'apprentissage

Une seconde rupture a lieu à l'entrée du second cycle de l'enseignement fondamental. Depuis une vingtaine d'années, l'arabe standard est devenu langue d'enseignement, ce qui pose problème pour l'apprentissage des matières scientifiques que les enseignants, bilingues, ont apprises en français pour la plupart d'entre eux et qu'ils doivent désormais enseigner dans une langue qu'ils maîtrisent moins bien.

Enfin, à l'entrée au lycée, la situation de l'arabe standard connaît un dernier paradoxe. Le système d'orientation des élèves vers les différentes sections des lycées est basé sur les résultats obtenus à la fin de l'enseignement fondamental $\mathrm{II}^{2}$. Il s'ensuit que les élèves qui ont les meilleures moyennes sont dirigés vers les sections scientifiques. Ainsi, les élèves des sections littéraires sont paradoxalement ceux qui obtiennent les plus mauvais résultats au cours d'arabe, alors que les élèves des branches scientifiques, pour lesquels il s'agit d'une matière secondaire, ont des résultats bien meilleurs.

7 Enfin, un certain nombre de difficultés des élèves tiennent à la fois à la nature des textes étudiés ainsi qu'à l'époque de leur production (celle des Abbassides). Pour les textes littéraires contemporains, poétiques ou narratifs, les élèves n'éprouvent pas de grande difficulté et prennent plaisir à les lire. La lecture spontanée, hors école, de textes arabes dépend essentiellement du milieu socio-familial, mais les ouvrages qui sont d'actualité attirent les élèves.

Horaires

\begin{tabular}{|c|c|c|}
\hline Horaires & Classes littéraires & Classes scientifiques \\
\hline $1^{\text {re }}$ année & 5 heures & 2 heures \\
\hline $2^{\mathrm{e}}$ année & 5 heures & 2 heures \\
\hline $3^{\mathrm{e}}$ année & 5 heures & 1 heure \\
\hline $\begin{array}{l}\text { Horaires de français } \\
\text { (pour comparaison) }\end{array}$ & 5 à 6 heures & 7 heures \\
\hline
\end{tabular}

\section{Programmes}

Avant 1994-1995, les programmes étaient « classiques ». Depuis cette époque, l'étude des textes littéraires au second cycle est basée sur la lecture méthodique, comme en France pour la langue maternelle. Le programme comprend des textes littéraires, classiques et modernes, complétés par un programme thématique ( l'homme et la nature ", par exemple). Les textes modernes portent sur des auteurs nationaux (Marocains), maghrébins (Tunisiens), et arabes (auteurs égyptiens et contexte palestinien). L'objectif n'est pas l'apprentissage de la langue à travers les textes, mais l'accès au sens du texte et à la culture arabe.

9 Le programme est subdivisé en cinq matières : étude de textes littéraires courts ou d'extraits, cours de langue, dissertation, étude d'une œuvre complète, thèmes, enfin, qui sont traités de manière transversale au travers des quatre activités précédentes. La production orale n'est pas objet d'apprentissage. 


\begin{tabular}{|c|c|}
\hline $\begin{array}{c}\text { Voici quelques exemples de thèmes transversaux, repris en étude de textes ou d'œuvres com- } \\
\text { plètes et qui servent de support à l'expression écrite ou au travail d'apprentissage linguistique : }\end{array}$ \\
\hline Les valeurs humaines dans le Coran & La recherche \\
\hline L'homme et la nature & Le progrès \\
\hline L'homme et l'amour & La modernité \\
\hline L'homme et la liberté & L'évolution de la littérature \\
\hline
\end{tabular}

\section{Euvres complètes}

\begin{tabular}{|l|l|}
\hline \multicolumn{1}{|c|}{\begin{tabular}{c}
\multicolumn{1}{|c|}{ re année } \\
Un commencement et une fin \\
Shéhérazade
\end{tabular}} & $\begin{array}{l}\text { Najib Mahfouz (Égypte, roman) } \\
\text { Taoufik El Hakim (Égypte, théâtre irréel) }\end{array}$ \\
\hline \multicolumn{1}{|c|}{$\mathbf{2}^{\mathbf{e}}$ année } \\
$\begin{array}{l}\text { La lanterne d'Oum Hachim } \\
\text { Le jeu de l'oubli }\end{array}$ & $\begin{array}{l}\text { Yahya Hiki (Égypte, réalisme social) } \\
\text { Mehamed Berrada (Maroc, autobiographie) }\end{array}$ \\
\hline $\begin{array}{l}\text { Feuilles } \\
\text { Le vent d'hiver }\end{array}$ & $\begin{array}{l}\text { Moubarek Rabii (Maroc, réalisme historique) } \\
\text { Abdellah Laroui (Maroc, autobiographie) }\end{array}$ \\
\hline
\end{tabular}

\section{L'évolution en cours}

Initiée depuis 1994, la rénovation de l'enseignement de l'arabe est perçue de façon différente par nos deux interlocuteurs. Pour l'un, la rénovation est un grand progrès, surtout perceptible au plan de la méthodologie. Il s'agit « d'apprendre à apprendre », le professeur doit être « un animateur, un évaluateur, un planificateur ».

11 Pour l'autre interlocuteur, l'évolution est très grande, mais pas perçue forcément de façon positive. Le programme actuel, déterminé par les textes officiels, a restreint la liberté de choix des enseignants qui étaient auparavant libres de choisir les textes ou les ouvrages sur lesquels ils voulaient travailler.

Autre difficulté pour les enseignants, c'est la séparation, depuis une dizaine d'années, entre l'enseignement de l'arabe et celui de l'éducation islamique. Alors qu'il y a une formation spécifique pour cette dernière matière, il n'y en a pas pour l'enseignement de la littérature arabe. Les professeurs d'arabe ont eu une formation traditionnelle, alors que l'optique actuelle est de baser les cours sur les apports de la linguistique moderne, de la narratologie, de la littérature contemporaine.

Actuellement, les professeurs les mieux formés sont ceux qui ont pris des contacts suivis avec leurs collègues nationaux professeurs de français et se sont ainsi initiés aux concepts de la linguistique moderne et aux pratiques de la lecture méthodique.

\section{Les manuels}

Les manuels actuels se différencient des anciens, mais ayant été élaborés par trois équipes différentes, ils n'offrent pas aux élèves une vision cohérente. 
Pour les classes littéraires, le manuel de $1^{\text {ère }}$ année secondaire (AS) a été élaboré par une équipe de Rabat-Kénitra, celui de $2^{\mathrm{e}} \mathrm{AS}$ par une équipe de Casablanca, celui de $3^{\mathrm{e}} \mathrm{AS}$ par une équipe de Fès. Ils n'ont pas les mêmes références: celui de $2^{\mathrm{e}} \mathrm{AS}$ est plus traditionnel, sans textes modernes, alors que ceux de $1^{\text {re }}$ AS et de $3^{\text {e }}$ As s'appuient davantage sur les apports de la linguistique. Les nouveaux manuels développent des activités autour du texte. Les médias ne sont pas pris en compte, ni par les manuels qui n'offrent pas de textes médiatiques, ni par les enseignants.

\section{Méthode d'approche}

16 L'étude se veut centrée sur l'accès au sens, mais dans la réalité concrète, c'est encore la méthode traditionnelle qui prime: l'enseignant part des "prérequis" liés à l'extériorité du texte (connaissance de l'auteur, de l'œuvre et de l'époque : histoire et société), puis procède ou fait procéder à une lecture à haute voix et, enfin, pose des questions à partir du manuel. Les méthodes pratiquées restent très traditionnelles, voire "mécaniques", la majorité des enseignants ne pratiquent pas les méthodes actives.

17 L'inspection souhaite mettre en place une véritable « lecture méthodique ", partant des attentes des élèves face au texte pour accéder au sens. Mais les enseignants ont de grandes difficultés à s'adapter à cette évolution, la résistance psychologique, qui s'appuie sur le poids des habitudes, étant confortée par l'absence de formation linguistique des professeurs.

18 La méthode aujourd'hui préconisée se veut basée sur le «structuralisme ». Observation du texte, émission d'hypothèses de sens, vérification des hypothèses, autour de trois niveaux d'analyse : lexical, sémantique (symboles, images...), pragmatique. L'inspection préconise en particulier la méthodologie proposée pour les textes français par Viala et Schmitt dans Savoir lire.

\section{L'évaluation}

19 L'évaluation porte essentiellement sur les «compétences d'écriture » (culturelle, méthodique, communicative).

Les exercices concernent la pratique de l'écrit (dissertation littéraire, technique du résumé et de la discussion). Il n'y a pas d'évaluation de l'oral. Les modifications de l'évaluation depuis 1994 portent essentiellement sur les outils : « on cherche à être plus objectif par la mise en œuvre de grilles de réponses ».

21 L'évaluation sommative se fait selon plusieurs modalités : réponses à des questions portant sur la découverte ou l'appréciation du texte, réponses à des QCM, participation au cours, préparations faites à l'avance par les élèves, complétées par les tests de contrôle continu. 


\section{NOTES}

1. Arabe classique, modernisé (langues des médias).

2. Niveau d'enseignement correspondant au niveau du collège en France.

\section{RÉSUMÉS}

L'arabe standard au Maroc a le statut de langue nationale. Toutefois, des difficultés surgissent dans son apprentissage en raison du contexte multilingue propre à ce pays, même si des évolutions en cours ont entraîné des progrès au niveau méthodologique.

INDEX

Index géographique : Maroc

\section{AUTEURS}

\section{MICHEL MASSACRET}

Chargé de mission, mission langues européennes, CIEP, Sèvres, France

ROGER JEANSOULIN

Chargé d'études, département langue française, CIEP, Sèvres, France 